\title{
Urgences
}

\section{N'ajustez pas vos hallucinettes}

\section{Jean-Pierre April}

Numéro 10, 2e trimestre 1984

Spécial fantasmes

URI : https://id.erudit.org/iderudit/025152ar

DOI : https://doi.org/10.7202/025152ar

Aller au sommaire du numéro

Éditeur(s)

Urgences

ISSN

0226-9554 (imprimé)

1927-3924 (numérique)

Découvrir la revue

Citer ce document

April, J.-P. (1984). N'ajustez pas vos hallucinettes. Urgences, (10), 21-28.

https://doi.org/10.7202/025152ar d'utilisation que vous pouvez consulter en ligne.

https://apropos.erudit.org/fr/usagers/politique-dutilisation/ 
JEAN-PIERRE APRIL

N'ajustez pas

vos hallucinettes 


\section{N'AJUSTEZ PAS VOS HALLUCINETTES *}

C'est long, 639 ans en plein vide, dans un vaisseau stationnaire, sans autres histoires que les péripéties programmées des hallucinettes...

Heureusement Lucien dormait la plupart du temps, même les yeux ouverts. Son jeu vidéo projetait ses effets spéciaux directement dans la pupille du vidéo-joueur; quand Lucien avait reçu ses hallucinettes, en 1984, sa vision des choses avait changé - forcément...

Rien de mieux que ce gadget pour oublier l'imminence de la catastrophe mondiale. Grâce aux hallucinettes, Lucien avait trouvé le moyen de faire un bond dans I'histoire pour parvenir intact dans une période plus paisible, après la Troisième Guerre.

Son projet anti-temporel n'était-il qu'une fuite intérieure? Maintenant la question n'avait plus d'intérêt; depuis deux ou trois cents ans, il avait oublié le sens de son expérience. Pour tromper sa profonde solitude, Lucien avait trouvé de joyeux copains; ses hallucinettes lui présentaient des personnages colorés qui lui tombaient dans l'oeil et lui pénétraient dans le cerveau, jusque dans les rêves.

Ses compagnons préférés semblaient sortis tout droit d'une BD hallucinée. C'étaient les Trois Petits Jambonnots, gros, gras, pâteux et pâlots, avec trois orifices comme des trous de nuit dans leur face de guimauve. II y avait aussi Fleur-de-Fer, étrange demi-entité encapée de mystère, qui ne laissait voir que sa tête de bourgeon chromée et ses trois griffes de poulet mécanique. Mais celui qui méritait le plus grand respect était le Doctor Sphock. Sérieux comme un pape, il veillait à la totale immutabilité de La Berlue, ce nouveau type de vaisseau à l'épreuve de la folie.

\footnotetext{
* Ce texte doit paraître dans la revue de science-fiction québécoise Imagine..., dans le cadre d'un numéro spécial - $n^{\circ} 21$ : "Imagitexte" - où les auteurs ont produit un texte pour "illustrer" des dessins de science-fiction.
} 
Ce cher Sphock avait tellement réfléchi sur la destinée de la psychonef que son crâne s'était légèrement dégarni et une brèche lui avait ouvert l'arête du nez. Parfois, au milieu de la nuit infinie, Lucien y voyait circuler des synapses comme des étincelles entre ses yeux brillants. Malgré ce léger défaut, Sphock avait fière allure: la sagesse imprégnait ses nobles traits et son regard intelligent scrutait infatigablement le fond de la spirito-sphère, à l'affût des pièges de l'entropie.

Les trois petits cochonnets avaient la mitraillette facile, l'être métallofloral possédait des serres d'égorgeur, et Sphock, en dépit de ses bras confectionnés avec des bouts de tuyaux, pianotait sur ses touches comme un virtuoso du clavier. Avec cette équipe du tonnerre, Lucien pouvait fermer l'oeil et dormir en paix.

II lui arrivait régulièrement de sommeiller dix ou douze mois, bien allongé au froid dans son scaphandre-à-sommeil qui régulait ses fonctions vitales et le nettoyait toutes les saisons. Mais un bon matin, après une année de sommeil agité, Lucien s'éveilla en voyant un vaisseau foncer sur lui. Sa sieste de 8760 heures l'avait quelque peu engourdi; il dut perdre une demi-journée avant de réagir. Des scènes de cauchemar l'obsédaient, refusant de retourner à la nuit. Lorsque Lucien appela Sphock, il était trop tard: I'appareil étranger bombardait La Berlue.

$$
\text { * * * }
$$

Les fantasmes avaient commencé à perturber ses rêves lorsqu'il avait modifié ses hallucinettes pour décrasser quelques neurones inutilisés, plus précisément depuis qu'il avait vu Les Trois Petits Jambonnots et le chauve-cochon: I'histoire d'un gros jambonnot ailé qui avalait les trois cochonnets pour bouleverser les grands espaces spirituels...

Lucien n'en dormait plus. Bien sûr le scaph-à-somme l'assommait, mais il voyait toujours ce mauvais ange porcin en train de faire la loi dans le vaisseau. Après tous ces mois de cauchemars, une sérieuse migraine avait éveillé Lucien. Et 
quand il vit cette grosse cochonnerie volante en train de faire jaillir la sève du crâne de Fleur-de-Fer, il crut perdre la tête.

Vite, il lui fallait trouver ses hallucinettes pour modifier cette vision détestable. Mais elles n'étaient visibles nulle part, et Sphock ne lui était d'aucun secours, occupé qu'il était à mâcher un rayon de feu jailli du néant, ou peut-être du cerveau malade de Lucien. Les bons Petits Jambonnots étaient disparus, sans doute dévorés tout rond par cette espèce de chauvecauchon qui s'excitait les ailes dans l'air raréfié.

Soudain, en portant ses mains tremblotantes à ses tempes douloureuses, Lucien toucha les branches de ses hallucinettes! Pendant des mois il avait donc dormi avec ce cochon nocturne! Pas étonnant que l'animal continue de le hanter à son réveil. Sans tarder, Lucien saisit le bouton de contrôle de ses hallucinettes, sur sa tempe gauche, et il le tordit méchamment pour effacer le jambonnot ailé.

Seul résultat: Lucien tordit la nuit!

Le cochon volant fondait sur lui, il colla son gros groin grognant sur ses hallucinettes, tel une ventouse qui lui aspirait toute velléité, et Lucien bascula dans le grand vide noir de la spirito-sphère...

$$
* * *
$$

\section{La vie vous ennuie?}

Vivez la nuit.

Pour des rêves plus nets,

Essayez les hallucinettes.

Dès qu'il vit cette pub vidéo, Lucien songea à décoller définitivement de ce qu'on appelait encore le réel. Les explications qui suivaient firent tomber toutes ses réticences.

\section{LA RÉALITÉ N’EXISTE PAS, PUISQUE CHACUN EN A UNE VISION DIFFÉRENTE. \\ LA SEULE RÉALITÉ EST DANS LA PERCEPTION DE}




\section{CHACUN. \\ DONC, EN CHANGEANT VOTRE VISION DU MONDE, VOUS MODIFIEZ VOTRE RÉALITÉ.}

Le truc semblait d'une simplicité aussi désarmante qu'efficace. Une paire de lunettes hallucinatives, apparemment opaques, dont les branches étaient terminées par de petits appareils qui envoyaient des ondes dans le cerveau.

Vous glissez une mini-cassette dans les hallucinettes, et des images vous pénètrent par l'oeil gauche pour atteindre I'hémisphère droit du cerveau, celui des fantasmes... tandis que l'oeil droit, derrière le verre teinté, continue de percevoir votre environnement.

Un verre pour chaque hémisphère: I'un pour voir l'extérieur, I'autre pour rêver l'intérieur; voilà qui favorisera votre équilibre.

Lucien ne pouvait plus se passer de ses hallucinettes. Sans elles, il avait l'impression d'être nu, vide, sans attrait pour lui-même. Paradoxalement, les verres noirs de ses lunettes devinrent une fenêtre ouverte sur son monde intérieur. Bientôt la vision de son oeil gauche devait contaminer l'autre...

Son projet essentiel: échapper à l'entropie. Tuer le temps qui tue. Le monde vieillissait mal, il courait à sa perte, mais Lucien conçut ses hallucinettes comme une machine à ne pas voyager dans le temps. Pendant que I'histoire continuerait d'évoluer vers le Cataclysme, Lucien se réfugierait dans un vaisseau immuable pour foncer à rebrousse-temps et se maintenir dans l'éternel présent, hors du fatidique continuum spatio-temporel...

Plus tard, si jamais l'évolution débouchait sur une quelconque oasis de paix, Lucien regagnerait le temps historique, il assumerait sa vie - et sa mort - pour apparaître devant les anthropologues de l'avenir, inchangé, témoignage vivant de la folie d'une époque invivable. 
Lucien avait plongé trop loin dans ses fantasmes; il s'imaginait qu'il pouvait enlever ses hallucinettes à volonté, mais il restait prisonnier de la coquille anti-entropique de La Berlue. Le projet avait si bien marché que Lucien I'avait oublié!

Seul le valeureux Sphock semblait saisir le sens de cette expérience, mais il devait rester derrière les hallucinettes de Lucien.

Non, l'aventure n'avait plus aucun sens, les hallucinettes hallucinaient! Pendant que Sphock mâchait du feu et que Fleur-de-Fer, les serres bioniques sur son foie malade, vomissait une sève bilieuse, le gros jambonnot flip-flappait comme un cochon de basse-cour en matraquant tout ce qui avait forme de crâne. Et le vaisseau rival, réel ou non, en profitait pour trouer la carapace de La Berlue.

Parce qu'il s'était endormi avec ses hallucinettes, Lucien était à la merci de ses visions. Bloqué dans l'anti-temps, figé comme une momie hors de son scaphandre, il tenait son pistolaser devant lui, incapable de régler la commande de ses hallucinettes. Impossible de les enlever, ou de remplacer le programme détraqué par une mini-cassette moins endiablée, bourrée de super-starlettes spatiales.

Autrefois Lucien avait abusé des programmes érotiques, mais lorsqu'il avait sélectionné les Trois Petits Jambonnots et que Sphock était entré dans sa vie, Lucien avait perdu sa liberté. Désormais Sphock avait son mot à dire, du moins quand on ne lui tirait pas du feu dans les dents.

- Docteur Sphock! suppliait Lucien dans son cerveau à demi paralysé, je vous en prie, venez régler mes hallucinettes.

Rapide et attentif, Sphock ne mit que quelques heures pour évaluer la situation. S'il ne prenait pas le contrôle du programme, le gros jambonnot et le vilain vaisseau enverraient valser La Berlue dans le continuum entropique. 
Vif comme une synapse, Sphock remonta les conduits électropsychiques des hallucinettes jusqu'au mécanisme de contrôle, collé à la tempe gauche de la victime. D'une poigne de fer, il tordit le bouton, la nuit et le cerveau de Lucien, jusqu'à ce que le vaisseau pirate jaillisse de l'oeil halluciné.

$$
\text { * * * }
$$

Tout va pour le mieux, maintenant que j'ai pris les commandes de La Berlue. Le gros cochon volant vient d'éclater en délivrant les Trois Petits Jambonnots, aussi dodus que des nounours en pâte. Mais j'ai dû cacher les hallucinettes de Lucien; à quoi bon lui rappeler un mauvais souvenir, surtout qu'il ne peut plus les utiliser. Les corsaires de la spirito-sphère lui ont crevé l'oeil gauche; ne reste qu'une cavité vide, bordée d'une tache écarlate. Clin d'oeil de sang.

- Doctor Sphock, me dit-il dans sa tête, je n'en peux plus d'être figé dans l'anti-temps; je veux vivre, laissez-moi mourir; je vous en suplie, éteignez mes hallucinettes.

II croit qu'il les a toujours... Sans doute parce que le programme des trois cochonnets lui est resté coincé dans le cerveau. Mais les jambonnots sont devenus craintifs, et Fleurde-Fer continue de crisper sa patte bionique sur sa poitrine; sa tête de bourgeon ne fleurira jamais.

Lucien ne peut que fixer son oeil unique droit devant lui, sur son destin immuable. Le plus souvent il croit voir un mur de mots qui lui voile l'horizon, comme une page imprimée refermée sur son monde; mais parfois le mur pivote pour laisser apparaître un être humain, énorme, toujours seul, qui vient interroger son regard vide, puis la page se referme sur sa nuit.

Autrefois, pendant les longs "voyages" dans les psychonefs, les membres d'équipage s'entre-tuaient. À l'arrivée, il n'y avait souvent qu'un seul survivant. Voilà pourquoi on a préféré les passagers uniques, accompagnés des personnages 
imaginaires des hallucinettes. Désormais l'explorateur spatiospirituel peut tuer tous ses fantasmes.

Mais pas moi!

Dissimulé dans le cerveau de Lucien, j'avais lu ses pensées avant même qu'elles soient complètement formulées. Depuis longtemps je savais qu'il voulait $m$ 'effacer pour revenir à ses starlettes spatiales. Mais j'ai profité de son sommeil pour dérégler les hallucinettes. Maintenant c'est moi qui commande La Berlue. Cap sur 2948, c'est ma seule issue.

Mais j'en ai déjà trop dit, car il s'agit de rester immobile pendant que le monde continue de mourir. Quand le mur de mots s'ouvre devant lui, ce sont peut-être des mortels que Lucien croit apercevoir, comme des fantasmes évanescents, pris dans l'entropie...

Pourvu que je puisse continuer de paralyser le bras de Lucien... Sinon je suis certain qu'il va porter son pistolaser contre sa tempe gauche, pour me tuer dans son cerveau. 